\title{
Comprehensive review on the pathophysiology, clinical variants and management of pemphigus (Review)
}

\author{
VICTOR-VLAD COSTAN ${ }^{1}$, CRISTINA POPA ${ }^{2}$, MĂDĂLINA FLORINA HÂNCU ${ }^{3}$, \\ ELENA PORUMB-ANDRESE ${ }^{4}$ and MIHAELA PAULA TOADER ${ }^{2}$ \\ Departments of ${ }^{1}$ Oral and Maxillofacial Surgery, and ${ }^{2}$ Oral Medicine and Oral Dermatology, \\ 'Grigore T. Popa' University of Medicine and Pharmacy, 700115 Iași; \\ ${ }^{3}$ Department of Dermatology, 'Sf. Spiridon' Clinical Emergency County Hospital, 700111 Iași; \\ ${ }^{4}$ Department of Dermatology, 'Grigore T. Popa' University of Medicine and Pharmacy, 700115 Iași, Romania
}

Received July 9, 2021; Accepted August 9, 2021

DOI: $10.3892 /$ etm.2021.10770

\begin{abstract}
Pemphigus represents a group of chronic inflammatory disorders characterized by autoantibodies that target components of desmosomes, leading to the loss of intercellular adhesion between keratinocytes and causing intraepithelial blistering. The pemphigus group consists of four main clinical types with several variants: pemphigus vulgaris (with pemphigus vegetans and pemphigus herpetiformis as variants), pemphigus foliaceus, paraneoplastic pemphigus and IgA pemphigus (with two clinical variants: intraepidermal neutrophilic IgA dermatosis and subcorneal pustular dermatosis). Genetic factors are involved in the pathogenesis, with HLA-DR4 (DRB1*0402) and HLA-DRw6 (DQB1*0503) allele more common in patients with pemphigus vulgaris, HLA class II DRB1*0344 and HLA Cw*1445 correlated with paraneoplastic pemphigus, and HLA-DRB1*04:01, HLA-DRB1*04:06, HLA-DRB1*01:01, HLA-DRB1*14, associated with a higher risk of developing pemphigus foliaceus. Autoantibodies are conducted against structural desmosomal proteins in the skin and mucous membranes, mainly desmogleins, desmocollins and plakins. Cell-mediated immunity may also play a role, especially in paraneoplastic pemphigus. Patients may present erythema, blisters, erosions, and ulcers that may affect the skin, as well as mucosal surfaces of the oral cavity, eyes, nose, leading to severe complaints including pain, dysphagia, and fetor. Oral mucosal postbullous erosive lesions are frequently the first sign of disease in pemphigus vulgaris and in paraneoplastic pemphigus, without skin involvement, making the diagnosis difficult. Treatment
\end{abstract}

Correspondence to: Dr Mădălina Florina Hâncu, Department of Dermatology, 'Sf. Spiridon' Clinical Emergency County Hospital, 1 Bulevardul Independenței, 700111 Iași, Romania

E-mail: madalinahancu92@yahoo.com

Key words: pemphigus vulgaris, pemphigus vegetans, pemphigus herpetiformis, pemphigus foliaceus, paraneoplastic pemphigus, IgA pemphigus options classically include immunosuppressive agents, such as corticosteroids and corticosteroid-sparing agents such as azathioprine, mycophenolate mofetil, cyclophosphamide, methotrexate or dapsone. Newer therapies focus on blocking cell signaling events induced by pathogenic autoantibodies and/or targeting specific autoantibodies. The disease evolution is conditioned by the treatment with maximum doses of corticosteroids and the side effects associated with long-term immunosuppressive therapy, which is why patients need a multidisciplinary approach in following the treatment. In this review, we provide a comprehensive overview of the epidemiology, pathophysiology, clinical aspect, diagnosis and management of the main intraepidermal blistering diseases from the pemphigus group.

\section{Contents}

1. Introduction

2. Research methods

3. Pemphigus group

4. Pemphigus vulgaris (PV)

5. Paraneoplastic pemphigus (PNP)

6. Pemphigus foliaceus (PF)

7. IgA pemphigus

8. Conclusions

\section{Introduction}

Pathophysiologically, intraepidermal blistering diseases represent a group of disorders in which the body wrongly attacks healthy tissue with autoantibodies that attach to structural proteins in the mucous membranes and skin, which are components of desmosomes (desmocollins, desmogleins, plakins), causing intraepithelial blisters (1-3). This category of intraepidermal blistering diseases includes pemphigus, which can be classified into the following four entities: pemphigus vulgaris (PV), pemphigus foliaceus (PF), paraneoplastic pemphigus (PNP), IgA pemphigus (subcorneal pustular dermatosis and intraepidermal neutrophilic IgA dermatosis). 
The specific symptoms and severity of these diseases vary from one person to another, even among individuals with the same disorder. The diagnosis of bullous skin diseases is based on the typical skin manifestations, which may be objectified by Nikolsky sign and characteristic direct immunofluorescence (DIF) patterns in skin biopsies (1-3). The presence of specific circulating autoantibodies guides the diagnosis and allows a correlation between the levels of specific autoantibodies and the severity of the disease (4).

Since the outbreak of the COVID-19 pandemic, oral ulcerative lesions have been described, associated with SARS-COV-2 infection. Bullous dermatoses can arise with similar lesions in the oral cavity, which is why the clinical picture must be very well known (2-4).

Although there is no cure for autoimmune blistering diseases, they can often be controlled with treatment. The choice of drugs and their dosage should be based on clinical severity and patient comorbidities. Most patients require months or even years of immunosuppressive maintenance therapy. In other cases, untreated autoimmune blistering diseases can cause life-threatening complications (1-5). In recent years, new insight into the causes and development of these disorders has led to research into new therapies, such as the development of drugs that target the specific autoantibodies that cause the symptoms of these diseases (6).

\section{Research methods}

A literature search was conducted, using electronic databases Key Elsevier, Medscape, PubMed, Google Scholar, for the term 'pemphigus' in combination with 'vulgaris', 'vegetans', 'herpetiformis', 'foliaceus', 'paraneoplastic', 'Ig A', 'epidemiology’, 'pathophysiology', 'skin manifestations', 'mucosal manifestations', 'clinical variants', 'management' and 'evolution' to collect reports of skin and mucosal manifestations described in patients with different clinical variants of pemphigus. Case reports, case series, and literature review-type articles were included in our research. A brief report was conducted based on 98 articles found in the literature.

\section{Pemphigus group}

Pemphigus includes a group of potentially life-threatening bullous autoimmune disorders of largely unknown etiology. Clinically, they are characterized by flaccid blisters and erosions of the skin and/or mucous membranes (1-4,7-9). The loss of intraepidermal adhesion between keratinocytes is attributable to the binding of autoantibodies directed against desmosomal structural proteins, primarily desmogleins (Dsg1 and Dsg3) and, in rare cases, also desmocollin 1-3 or plakins $(1-4,10)$. Pemphigus has distinct forms: pemphigus vulgaris (PV), pemphigus foliaceus (PF), paraneoplastic pemphigus (PNP), and IgA pemphigus. $\mathrm{PV}$ and $\mathrm{PF}$ are caused by a humoral autoimmune response, whereas PNP is caused by both humoral and cellular autoimmune responses (11). PV is characterized by persistent mucosal erosions with or without skin involvement. PF presents fragile, superficial blisters, as well as subsequent erosions and leafy scales that exclusively affect keratinizing skin (11). In PNP, the clinical hallmark is painful oral mucosal lesions accompanied by morphologically heterogeneous skin lesions (erythematous macules, flaccid blisters, scaly plaques, or erosions) $(4-8,11)$.

\section{Pemphigus vulgaris (PV)}

Epidemiology. According to several retrospective studies, pemphigus vulgaris (PV) is the most frequent representative of the group of pemphigus diseases, with an incidence of $0.1-0.5 / 100,000$ population (7-9,11). A female predominance is reported in most epidemiological studies, with a peak age between 50-60 years, although childhood onset forms have been described $(7,12)$. PV is also more common in certain ethnic groups, such as the Ashkenazi Jewish population and Mediterranean descendants $(7,12)$.

Pathophysiology and genetic factors. In patients with PV, most types of antibodies are oriented against desmosomal cadherins, Dsg1 and Dsg3, but other autoantibodies have been identified targeting other metabolic and structural proteins, such as Dsc1 and Dsc3 desmocolins, mitochondrial antigens, hSPCA1, thyroid peroxidase, muscarinic and nicotinic acetylcholine receptors, plakoglobin, E-cadherin and plakophilin 3 (9,12-14). The pathogenic role of these non-Dsg autoantibodies is mentioned by some studies, which suggest that they synergistically complement the classic effects of anti-Dsg autoantibodies in the complex process of pemphigus pathogenesis (14). The two antigens targeted by autoantibodies in PV are the $130-\mathrm{kDa}$ glycoprotein Dsg3 and $160-\mathrm{kDa}$ glycoprotein Dsg1. Dsg1 is mainly expressed on the surface of the epidermis, while Dsg3 accumulates predominantly in the mucous membranes and deeper epidermal layers (9-13). Patients with mucosal-dominant-type PV have only anti-Dsg3 antibodies, and those with mucocutaneous-type PV have both anti-Dsg3 and anti-Dsg1 antibodies (13).

There is a genetic predisposition for developing PV; certain major histocompatibility complex (MHC) class II molecules, such as DR4 (DRB1*0402) and DRw6 (DQB1*0503) occurring more frequently among those affected $(7,15)$. Although these alleles are rare in the European population, they are more common in certain ethnic groups (e.g. Jewish population) and in countries of the eastern Mediterranean and the Middle East (Turkey, Iran, Iraq) (4-7,15).

Clinical features. PV is clinically characterized by flaccid blisters/erosions of the mucous membranes and the skin.

In most of the cases, the onset of the lesions involves the oral cavity. It is often not recognized in the early stages, thus, other oral ulcerative disorders are suspected, such as herpetic gingivostomatitis, recurrent aphthae, erosive oral lichen planus, or even candida stomatitis. Intact bullae are rare in the mouth. More commonly the lesions are ill-defined, irregular, painful erosions located on the gingiva, buccal or palatal mucosa $(8,16-18)$. Other sites of the mucous membrane may be affected, including the conjunctiva, esophagus, pharynx, larynx, urethra, penis, labia, vagina, cervix, and anus (8) (Fig. 1).

Skin lesions appear several weeks or months after the onset of mucosal erosions and may develop anywhere on the skin, but there are some areas of predilection that include the scalp, face, chest, axillae, groin, and umbilicus. Blisters are 


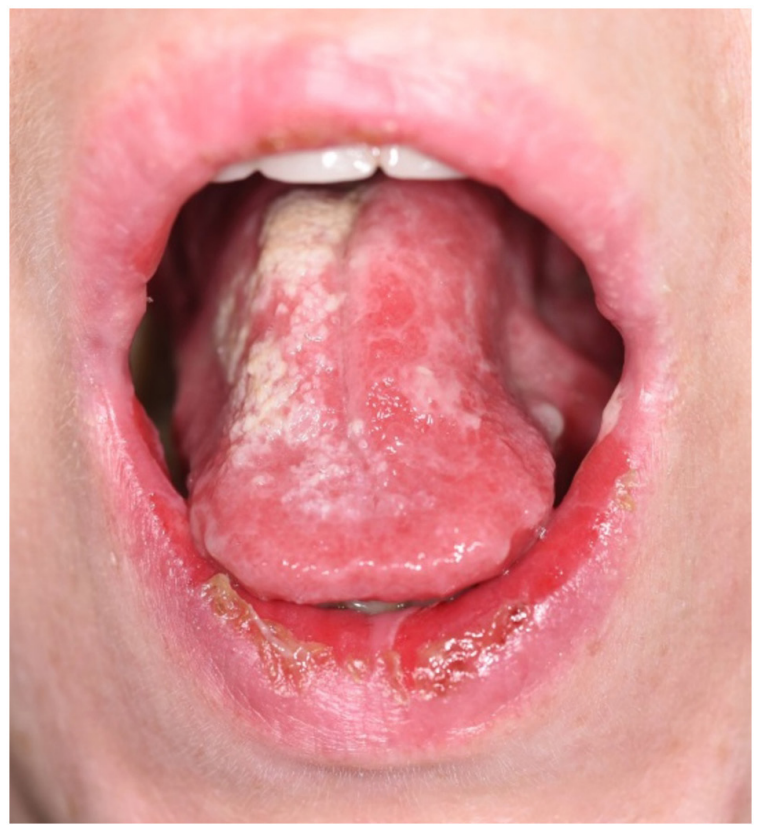

Figure 1. Clinical manifestation of mucosal pemphigus vulgaris.

flaccid, fragile and break easily, leading to painful erosions, which bleed easily and often become crusted, and can lead to residual pigmented lesions after healing under immunosuppressive treatment (5,6,8-10) (Fig. 2). The blister on the skin may remain localized for 6 to 12 months, and then afterwards becomes widespread. The lesions can be painful, pruritic, and associated with a burning sensation, weakness, history of epistaxis, malaise, weight loss, dysphagia, and hoarseness $(4,7,8)$. It is uncommon that the lesions emerge as a generalized acute eruption (9). During the active phase of PV, Nikolsky signs can be obtained but they are not specific to PV and can be found in other active blistering diseases (8). The direct Nikolsky appears because of an absence of cohesion within the epidermis and its upper layers move easily laterally with slight pressure or rubbing. Another sign that may be present is Asboe-Hansen sign also referred to as the 'indirect Nikolsky' or 'Nikolsky II' which occurs when a gentle pressure on intact bulla forces the fluid to spread under the skin away from the site of pressure -'bulla-spread phenomenon' $(1,3,4-8)$. In the case of pregnant women with active pemphigus, there is a chance that the newborn could develop neonatal pemphigus as a result of the transmission of maternal IgG (consisting of autoantibodies against Dsg3) through the placenta $(8,19)$. The clinical picture in neonatal pemphigus is not as severe compared to the disease that caused it since it is not a systemic disease. The symptoms and signs are reduced to skin lesions, and exanthematous; crusted erosions erupt as a temporary phenomenon over several weeks until the degradation of maternal autoantibodies (19-21). Neonatal pemphigus has a good prognosis (19). In addition to classical PV, other special forms exist (8). Pemphigus vegetans is characterized by the tendency to develop papillomatous and verrucous intertriginous vegetations $(8,22,23)$. Most patients initially present to their health care provider with stomatitis or hyperkeratotic plaques in a cerebriform pattern on the tongue (23). The cutaneous lesions rupture

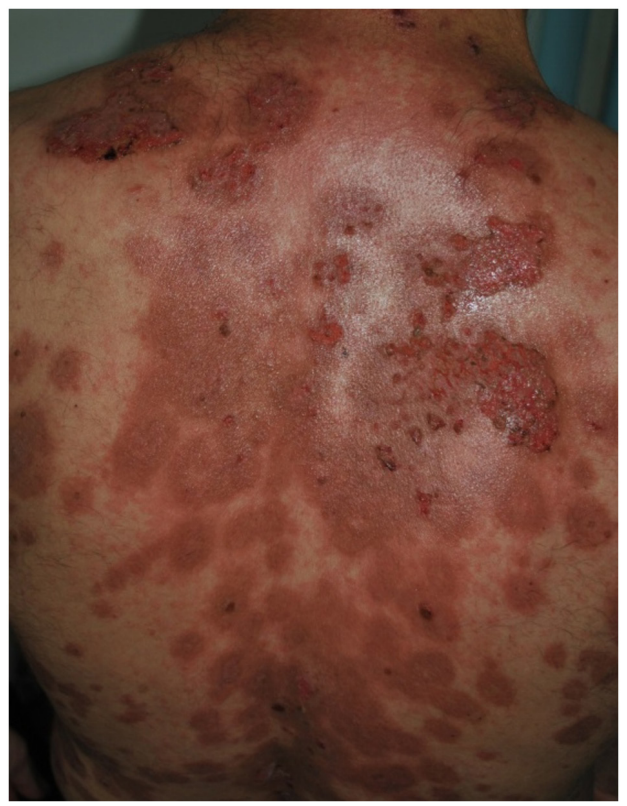

Figure 2. Cutaneous manifestations of pemphigus vulgaris. Erosions with serous and hemorrhagic crusts, localized on seborrheic areas of the trunk.

and ulcerate with verrucous crusting and vegetative plaques forming over the erosions. These hyperkeratotic lesions characteristically present in the intertriginous areas including the groin/inguinal folds, armpits, thighs, nasolabial and flexural surfaces (Fig. 3) $(8,23)$. Depending on the clinical course, two subtypes of pemphigus vegetans are differentiated. The Neumann subtype is characterized by large vesiculobullous and erosive lesions, with an aggressive course by the formation of whitish, macerated plaques. In the Hallopeau subtype, pustules initially appear which later turn into warty lesions and has a more indolent course (8,23-25). Untreated, pemphigus vegetans can be fatal within 5 years due to severe blistering, secondary infection and malnutrition. Mortality is approximately 5 to $15 \%$ per year $(8,22-24)$.

Pemphigus herpetiformis is a rare variant of $\mathrm{PV}$, characterized by erythematous, vesicular, bullous, pustular or papular lesions, often in a 'herpetiform' pattern and with severe pruritus, frequently located on the trunk and proximal extremities $(8,26)$. Skin lesions tend to present with annular-shaped distribution, or in some cases, the main lesions can resemble urticaria $(26,27)$. Oral mucosa involvement is rare $(8,27)$. Therefore, pemphigus herpetiformis possesses clinical similarity to dermatitis herpetiformis and must be included in the differential diagnostic considerations (27).

Diagnosis. PV diagnosis is based on a combination of clinical presentation (presence of recurrent blister formation, erosions and crust, Nikolsky sign); histological detection of intraepidermal blistering; detection of acantholytic keratinocytes by the Tzanck test; detection of pemphigus antibodies, DIF, IDIF, ELISA (9-12).

Histopathological examination will reveal acantholysis and a sparse inflammatory infiltrate. The acantholysis occurs in the suprabasal layer, leaving a single layer of basal keratinocytes attached to the dermal-epidermal basement membrane looking like a 'row of tombstones' (4,7,9-12). In the early 


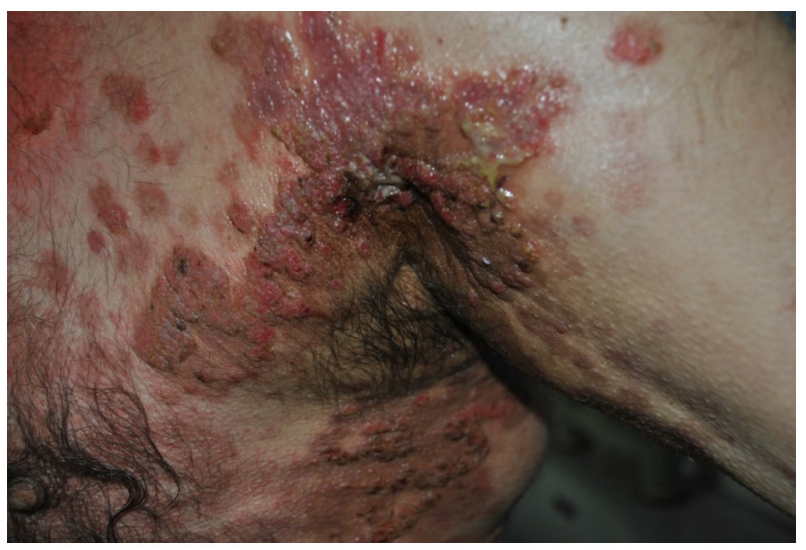

Figure 3. Cutaneous manifestations of pemphigus vegetans. Papillomatous and verrucous vegetations localized in the intertriginous areas.

pemphigus vulgaris. pre-bullous stage, histology also shows eosinophilic spongiosis (12).

Direct immunofluorescence (DIF) of patients' perilesional skin reveals a reticular fluorescence pattern, a 'honeycomb-like pattern', caused by the deposition of IgG autoantibodies and C3 on the surface of epidermal keratinocytes (4,28-30). The detection of circulating $\operatorname{IgG}$ autoantibodies can be conducted using methods such as indirect immunofluorescence (IIF), using monkey esophagus or human skin as the substrate (29-31). ELISA and chemiluminescent enzyme immunoassay using recombinant Dsgs enable detection of circulating autoantibodies in pemphigus (12,32-34). Currently, there is no consensus on which assay should be used as a diagnostic test for PV, but ELISA is one of the most accurate diagnostic tests, separately measuring anti-Dsg1 and anti-Dsg3 IgG. In a meta-analysis of 13 studies with a sample size of 1,058 patients, anti-Dsg3 ELISA demonstrated a sensitivity of $97 \%$ and specificity of $98 \%$ in PV (34). ELISA or chemiluminescent enzyme immunoassay are useful for both diagnosis and monitoring of disease activity, as autoantibody titers often fluctuate in parallel with disease activity and decrease with clinical improvement (7,32-34). In 20 to $40 \%$ of patients, even after clinical remission, anti-Dsg1 and anti-Dsg3 autoantibodies remain detectable and they are occasionally detectable in clinically healthy individuals $(7,35)$.

There are three subtypes of PV measured by the pattern of autoantibodies: mucosal-dominant PV, when serum is positive for anti-Dsg3 but negative for anti-Dsg1; mucocutaneous PV, when serum is positive for anti-Dsg3 and anti-Dsg1 and show the implication of the epidermis in addition to the mucous membranes; cutaneous PV is scarcely and correlated with blistering in deep epidermal layers due to anti-Dsg1 and pathogenically weak anti-Dsg3 (12,28-31). In patients suffering from PV, many autoantibodies have been found to aim at other structural and metabolic proteins, including desmocollins (Dsc) 1 and 3, muscarinic and nicotinic acetylcholine receptors, mitochondrial antigens, thyroid peroxidase, hSPCA1, plakophilin 3, plakoglobin, and E-cadherin (14,31,35-37). Research on some of these non-Dsg autoantibodies implies that they complement the typical effects of anti-Dsg autoantibodies in pemphigus pathogenesis $(14,37)$. A cross-sectional study and meta-analysis reported a high incidence of other coexisting autoimmune disease of patients with PV, such as thyroid diseases (e.g hypothyroidism), rheumatoid arthritis, type 1 diabetes mellitus, inflammatory bowel disease, alopecia areata, vitiligo, systemic lupus erythematosus, scleroderma, and rare entities such as myasthenia gravis $(38,39)$. As part of PV investigations and surveillance, investigation for these conditions should be considered.

Treatment. There is high morbidity and mortality within the population suffering from PV. Systemic corticosteroids and immunosuppressants remain the main therapy and have managed to decrease the mortality rates from 75 to $10 \%$. The purpose of initial therapy is to control the disease by minimizing the blister formation, stimulating the healing of present blisters, and prolong remission with a minimum dose (e.g. oral prednisolone $\leq 0.2 \mathrm{mg} / \mathrm{kg} /$ day or $\leq 10 \mathrm{mg} /$ day) $(7,12,40)$.

The latest guidelines recommend corticosteroids $(0.5-1.5 \mathrm{mg} / \mathrm{kg} / \mathrm{day})$ as the first treatment in the initial phase. Steroid-sparing immunosuppressants can be added in case there is a high risk of an adverse reaction to CS $(9-12,40)$. Systemic corticosteroids are reduced in concordance with the therapeutic response. Clinicians should be mindful of complications, eventually relapses, of long-term CS therapy during the maintenance period, in the likes of susceptibility to infections and infestations, osteoporosis, secondary adrenal insufficiency, hypertension, posterior subcapsular cataract, and transient hyperglycemia (41). During the steroid tapering phase around half of the patients relapse, and the other half reach complete remission after approximately 3 years of treatment $(40,41)$

Multiple immunosuppressive adjuvants have been used to lessen the complications of high doses of CS in the long-term. These include: azathioprine (AZA) with a recommended dose of $2.0 \mathrm{mg} / \mathrm{kg} /$ day with normal thiopurine methyltransferase activity and $1 \mathrm{mg} / \mathrm{kg} /$ day with TPMT enzyme mutations; cyclophosphamide (CYP) $2 \mathrm{mg} / \mathrm{kg} /$ day, i.v. pulse therapy or continuous oral administration; mycophenolate mofetil (MMF) $2 \mathrm{~g} /$ day, divided into two doses; methotrexate (MTX) 10-20 mg/week; dapsone, but before the administration a measuring of serum glucose-6-phosphate dehydrogenase (G6PD) activity is mandatory $(7,42,43)$.

Patients who are unable to reach clinical remission with systemic CS and/or immunosuppressant agents, or who present moderate to severe pemphigus or refractory $\mathrm{PV}$, might undergo high-dose intravenous immunoglobulins (IVIg) treatment, plasmapheresis, or extracorporeal immunoadsorption (IA) (7,12,44-46). IVIg treatment is well tolerated, mostly safe, and works by quickly decreasing the autoantibodies which are responsible for pemphigus, targeting pathogenic antibodies (10-12,44-46). Plasmapheresis is effective especially when it is combined with immunosuppressant agents (e.g. pulsed IV cyclophosphamide), and numerous clinical trials have indicated the increased efficacy and blistering diminishing with this treatment $(7,9-12,45)$. IA uses affinity adsorption of pathogenic autoantibodies. These autoantibodies attach to the adsorber through an immobilized ligand. A quick and substantial decline in desmoglein (Dsg)-reactive autoantibodies, along with clinical remission of mucocutaneous erosions and blisters has been observed when applying IA in severe PV. Systemic immunosuppressive medication 
can be combined with IA and is safe and well-tolerated in general $(10,11,46)$.

Recently, targeted biologic therapies have been adopted in pemphigus, such as rituximab (RTX) and tumor necrosis factor (TNF)- $\alpha$ inhibitors (47). Rituximab is a chimeric type I anti-CD20 monoclonal antibody, which can bind to CD20 antigen and remove B-lymphocytes expelling CD20 from blood $(7,12,48)$. Rituximab leads to a clear reduction of circulating anti-Dsg autoantibodies at the expense of B cells, and because of the pathogenic role of these autoantibodies, there is a noteworthy amelioration of the lesions $(12,47-49)$. Initial treatment with RTX, in combination with high potency topical CS or IVIg, has shown to be efficient in patients with pemphigus that have a contraindication to systemic steroids (47-49).

A recent clinical study involving 11 patients that had PV refractory to conventional therapy showed that three weeks of treatment with RTX (375 mg/m²/week) followed by IVIg $(2 \mathrm{~g} / \mathrm{kg}$ ) for four weeks, and then four consecutive months with monthly infusions of IVIg and RTX, resulted in remission in 9 of the patients which lasted from 22 to 37 months (47-50). Another study with 136 patients suffering from refractory pemphigus coming from 4 European countries reported a $95 \%$ average response rate, with $2 / 3$ of patients achieving complete remission (51). The most common side effects of RTX include infections and adverse events related to infusion. Opportunistic infections may also arise, including cytomegalovirus and pneumocystis jirovecii infections and theory describes the risk of hepatitis $\mathrm{B}$ and $\mathrm{C}$ virus reactivation, as well as tuberculosis (52-54). Late reactions include vasculitis, hypersensitivity (serum sickness), Steven-Johnson syndrome and some cases have shown paradoxical pemphigus flares consequent to RTX treatment (55). RTX has revolutionized PV treatment, but some patients remain refractory to this agent and for such refractory cases, new drugs are being tested in clinical trials. Ofatumumab is a fully humanized anti-CD20 mAb, which is less immunogenic than RTX (56). Veltuzumab is a humanized anti-CD20 mAb that can be administered subcutaneously and shows clinical efficacy in patients with refractory PV. Veltuzumab is a more economical alternative to intravenous RTX, because a lower dose is required (57).

For the treatment of oral lesions, intralesional RTX was reportedly effective in 3 patients with PV with oral lesions refractory to systemic therapy, including intravenous RTX (58).

Evolution and complications. Most deaths associated with untreated PV occur within the first few years of the disease onset. Considering that the drugs used in the treatment of PV have serious side effects, patients must be monitored carefully for infections, liver and renal function abnormalities, electrolyte disturbances, osteoporosis, hypertension, diabetes, anemia, and gastrointestinal bleeding (7,10-12).

\section{Paraneoplastic pemphigus (PNP)}

Epidemiology.PNP represents a rare disorder with an incidence and prevalence that remains unclear. There are $\sim 500$ cases reported in the literature, and PNP accounts for 3-5\% of all pemphigus cases $(7,12)$. Patients between 45 and 70 years of age are usually affected, and a female predominance is reported in most epidemiological studies $(7,9-12,59)$. However,
PNP can affect also children and adolescents, particularly in association with Castleman's disease. Clear racial, ethnic, or geographic differences in the risk for PNP have not been established $(7,9-12,59)$.

Pathophysiology and genetic factors. The etiopathogenesis of PNP is not completely known, but it is plausible that both autoantibodies and cell-mediated immunity play a key role.

The most common autoantibodies detected in PNP are directed against the plakin family, such as envoplakin (210-kDa), periplakin (190-kDa), bullous pemphigoid antigen I (230-kDa), desmoplakin I (250-kDa), desmoplakin II (210-kDa), plectin (500-kDa), and $\alpha 2$-macroglobulin-like-1 (170-kDa) $(7,12,59)$. Tsuchisaka reported that epiplakin is a PNP autoantigen, being detected in 35 (72.9\%) of 48 PNP sera of Japanese patients by immunoprecipitation-immunoblotting (60). PNP antibodies are typically $\operatorname{IgG}$, although $\operatorname{Ig}$ A has been reported in a few cases (59-61).

In a review, Czernik et al summarized that cell-mediated immunity may also play a role in PNP, highlighting lesional mononuclear cells and elevated IL-6 levels in the sera of patients with PNP (61). In addition, Wade and Black detected MHC-restricted $\mathrm{CD}^{+}$cytotoxic T cells, non-MHC-restricted $\mathrm{CD}^{+} 6^{+}$, and $\mathrm{CD}_{68}^{+}$natural killer cells within the dermoepidermal junction of PNP lesions (62).

Regarding the genetic predisposition, an association with HLA class II DRB1*0344 and HLA Cw *1445 confer strong susceptibility to PNP in Caucasian and Han Chinese patients. These conclusions were drawn by Martel et al (63) from a series of 13 Caucasian French patients.

Clinical features. Clinical features are extremely polymorphous in PNP, and lesions can be detected not only on the skin, but also on different mucosae. The cross-reactivity with tumor antigens and the presence of different autoantibodies could justify the different manifestations in PNP patients $(59,62-64)$. PNP can be the first clinical manifestation that leads to the detection of an occult tumor in $\sim 30 \%$ of cases $(7,12,59)$. PNP is associated with underlying neoplasms and the most frequent include non-Hodgkin's lymphoma (38.6\%), chronic lymphocytic leukemia (18.4\%), Castleman's disease (18.4\%, benign tumors, commonly in children), adenocarcinomas (prostate, pancreas, breast, gastric), squamous cell carcinomas (8.6\%), sarcomas $(6.2 \%)$, thymoma (5,5\%), Waldenström's macroglobulinemia (1.2\%), Hodgkin lymphoma (0.6\%), and monoclonal gammopathy (0.6\%) (12,59,62-64).

Initially, PNP typically manifests as hemorrhagic stomatitis with extensive mucous membrane erosions accompanied by intense pain and resistance to therapy $(64,65)$. The lesions are polymorphic, and symptoms such as blisters, erosions, spots, papules, and plaques can occur, involving the lips, vermilion and the tongue (62-65). Painful erosions and crusting on the lips could resemble oral lesions commonly found in erythema multiforme (EM) or Stevens-Johnson syndrome (59). In children, the stomatitis caused by PNP may be often mistaken for herpetic stomatitis or toxic epidermal necrolysis (TEN), leading to a delay in the diagnosis (66).

In addition to stomatitis, mucositis involving the pharynx, larynx, esophagus, and anogenital region can occur. Symptoms 
of oropharyngeal involvement may include a sore throat and dysphagia. Ocular involvement occurs in $\sim 70 \%$ of cases and the most common symptoms and signs include painful ocular irritation, worsening of vision, mucus discharge, conjunctival erosions, eyelid margin thickening, corneal erosions, and pseudomembranous conjunctivitis. In several cases, mucosal involvement is the only sign of PNP $(38,39,65-67)$.

Skin lesions of PNP are polymorphic and usually appear after the onset of mucosal lesions, involving any site, but especially the torso, head, neck, and proximal extremities (59,62-64). Blisters and erosions are commonly observed and mimic those of PV,PF or bullous pemphigoid, affecting any area of the body, but especially the upper trunk. The erythematous maculopapular lesions with dusky centers or central vesicles may arise on the extremities, mimicking the erythema multiforme-like targetoid lesions (59,64-67). Another type of characteristic cutaneous lesions is represented by lichenoid eruptions, which manifest as erythematous papules or plaques, similar to that in lichen planus and graft-versus-host disease and are frequently identified in children, predominantly on the torso and limbs $(59,62,66)$. In some cases of PNP, cutaneous lesions may present as a nail or periungual lesions (onychodystrophy, erosions, scaling) and alopecia (59).

As for extracutaneous lesions, the involvement of the respiratory epithelium is frequently associated with pulmonary disease in the form of bronchiolitis obliterans, a frequently lethal obstructive respiratory disorder $(59-62,66)$. The initial symptom of bronchiolitis obliterans is dyspnea, and pulmonary function tests show obstructive lung disease. Bronchiolitis obliterans is found in $\sim 30 \%$ of PNP patients and frequently develops in patients with Castleman disease (65-67). Due to the involvement of diverse organ systems, PNP has recently often been viewed as a mucocutaneous variant of the 'paraneoplastic autoimmune multiorgan syndrome’ (PAMS) (61).

Diagnosis. The diagnostic criteria for PNP include different criteria, based on the clinical picture, histopathology, direct and indirect immunofluorescence and immunoprecipitation.

The clinical presentation includes painful erosions involving mucosae with or without a multiform skin eruption producing blisters and erosions, occurring in association with an occult or evident neoplasm.

PNP has two major clinical phenotypes, blisters and lichenoid eruptions, and depending on the type of lesion biopsied, the histological findings are variable, and often the diagnosis requires multiple biopsies (62-64). In blisters, suprabasal acantholysis and individual keratinocyte necrosis with sparse inflammatory infiltrate are observed, while in lichenoid eruptions, an interface and lichenoid dermatitis with a dense band-like lymphocytic infiltrate in the upper dermis are usually detected $(59,68)$. Sometimes, blisters and interface dermatitis may coappear in the same lesion (59). Histological findings show the combination of blisters in the epidermis caused by $\operatorname{IgG}$ autoantibodies (humoral autoimmune response) and interface dermatitis caused by self-reacting $\mathrm{T}$ cells (cellular autoimmune reaction) $(59,64,68)$.

DIF reveals IgG and complement $\mathrm{C} 3$ deposition both in the intercellular space and in the dermoepidermal junction, along the basement membrane zone (28-31,59-62). In the classic forms of pemphigus, IIF is positive only on stratified squamous epithelial substrates, but in PNP there is staining of other tissues, such as the bladder, heart, and liver $(28-31,59)$. IIF shows the presence of circulating IgG autoantibodies that target the intercellular proteins found in transitional or stratified squamous epithelia $(28-31,59)$.

Although immunoprecipitation is still the gold standard for the demonstration of specific autoantibodies, immunoblotting is a valuable aid for diagnosis (69). Immunoblot analysis using epidermal extracts has been used to detect $210-\mathrm{kDa}$ envoplakin and 190-kDa periplakin, which are highly sensitive and specific for PNP $(30,59,69)$. Immunoprecipitation can detect antibodies against multiple epidermal antigens, including desmoplakin I (250 kDa), bullous pemphigoid antigen (230 kDa), envoplakin (210 kDa), desmoplakin II (210 kDa), periplakin (190 kDa) and 2-macroglobulin-like-1 (170 kDa) (28-31,59-61).

Enzyme-linked immunosorbent assays (ELISAs) are a useful technique for detecting circulating autoantibodies in PNP, especially those against Dsgs and Dscs. Approximately $80 \%$ of patients with PNP have circulating anti-Dsg3 IgG; in $19-42 \%$ of patients, autoantibodies have been detected against other desmosomal cadherins (Dsg1, Dsc1, Dsc2, Dsc3), and in $40 \%$ of patients, ELISA reveals autoantibodies against BP180 $(34,59,64)$.

When PNP is suspected in a known patient with a history of malignancy, thorough investigations such as blood cell count, lactate dehydrogenase, flow cytometry, computed tomography of the chest, abdomen, and pelvis should be performed. Up to a third of the patients with PNP, have an underlying malignancy discovered after the onset of PNP symptoms (7,12,59-61).

The differential diagnosis of PNP includes pemphigus vulgaris, mucous membrane pemphigoid, erythema multiforme, Stevens-Johnson syndrome, toxic epidermal necrolysis, major aphthous stomatitis, oral lichen planus, graft-versus-host disease, and herpes simplex virus infection (59,62-64). In pediatric cases, oral manifestations may be mistaken for a herpetic stomatitis (66).

Treatment. The rarity of PNP makes it more difficult to treat. Even though some therapies have been proposed in the literature, PNP has been observed to be more resilient to treatment compared to different forms of pemphigus (59,62-64,65-67). If there is suspicion of PNP, the six steps reported by Frew and Murrell (70) should be followed for better management of the patient. The six steps are as follows: vital parameters stabilization, evaluation of any underlying malignancy, correct diagnosis of PNP, the extirpation and medical therapy of the trigger tumor, and PNP treatment using immunosuppression, immunomodulation, or plasmapheresis $(59,71)$.

Cases that are associated with benign tumors, such as benign thymoma and localized Castleman's disease, generally ameliorate or reach complete remission after complete tumor resection $(59,64)$. In patients with PNP and malignant neoplasms, extirpating the tumor does not lead to controlling the disease, and an agreement on the best treatment has yet to be recognized (64).

The first line of PNP treatment is a high dose of systemic corticosteroids (prednisolone), but many patients do not appear to have a good response with only corticosteroids (59,64-67,71). Corticosteroids only improve skin lesions, while mucosal lesions are resistant to most types of therapy. Steroid-sparing 
agents, namely cyclosporin, azathioprine, cyclophosphamide, and MMF, can be used with glucocorticoid therapy to lessen the total steroid burden $(62,64,71)$. IVIg and plasmapheresis manifest high efficiency, safety, and promising effects in the treatment of PNP (71). A $2 \mathrm{~g} / \mathrm{kg}$ dose per cycle is used for IVIg; these cycles are repeated monthly. IVIg act by reducing pathogenic autoantibodies rapidly. In addition, IVIg can be added to the patient's existing treatment regimen without the added concern of additional immunosuppression (44,62-64,70).

Alternative therapies are being applied notably in patients whose malignancy is in remission. RTX and ibrutinib are B-cell-targeting agents and they generate different outcomes among patients suffering from PNP associated with B-cell malignant lymphomas (70,71). Overall the efficiency of RTX in PNP is much less consistent than PV and PF. RTX is generally well tolerated; however, adverse effects include infusion and allergic reactions $(59,64,47-49)$.

According to reports, alemtuzumab, a humanized monoclonal antibody against CD5, which is shown in most B and T lymphocytes, has induced long-term remission in a patient with B-cell chronic lymphocytic leukemia. Alemtuzumab administered $30 \mathrm{mg}$ i.v., 3 times a week for 12 weeks, showed recovery of cutaneous and mucosal lesions (72). In two cases of PNP, tocilizumab, a monoclonal antibody anti-IL-6 receptor, was shown to quickly improve mucositis, although bronchiolitis obliterans did not shown signs of improvement (73).

Due to the risk of sepsis followed by iatrogenic immunosuppression and loss of skin integrity, early antimicrobial therapy is suggested. In the case of pain caused by extensive erosions, antalgic therapy could be beneficial (64,69-71).

Evolution and complications. The prognosis of PNP is generally poor, and the mortality rate ranges from 75 to $90 \%$, with a 5-year overall survival rate of only $38 \%$. Death is usually due to systemic complications, including sepsis, gastrointestinal bleeding, bronchiolitis obliterans and severe infection due to immunosuppressive therapy. Similar to mucositis, bronchiolitis obliterans is resistant to therapy, and lung transplantation is the last therapeutic option for respiratory failure $(59,62-64,67)$.

The evolution of PNP is not correlated with that of the associated malignancy. PNP lesions may progress after removal of the triggering malignancy or when the malignancy is under control. However, in patients with PNP and Castleman's disease or benign thymomas, the outcome is favorable after tumor removal (61). Paraneoplastic pemphigus may precede the clinical appearance of a neoplasm, which makes screening of these patients mandatory $(62,64-67)$.

The prognosis of PNP depends on a prompt diagnosis and early initiation of treatment. Effective control of the oral and skin lesions, proper treatment of the underlying neoplasm, and prevention of bronchiolitis obliterans are of paramount importance (70).

\section{Pemphigus foliaceus (PF)}

Epidemiology. PF is rare and sporadic worldwide and the incidence varies depending on the population studied.

In Western Europe, the incidence of PF is $\sim 0.5-1$ case per million per year. In South America (Brazil, Colombia, and
Peru) and North Africa (Tunisia and neighboring countries), the incidence of PF is higher than in other countries and this is because of an endemic form of the disease (fogo selvagem), which affects mostly young adults (4-7,9-12,74). In Brazil, the incidence of endemic PF in the Terena reservation is around $3.4 \%(7,12,74)$. The prevalence of PF in men and women is approximately equal, but in some regions such as the Sousse region of Tunisia, women are more affected (6.6 cases per million per year) (9-12,75). In El Salvador, a similar female and age predisposition may also be evident.

The mean patient age at onset of $\mathrm{PF}$ is $~ 50-60$ years, but it may occur at any age. Fogo selvagem often occurs in children, young adults, and genetically related family members, and the mean patient age at onset is $\sim 20-30$ years $(4-7,9-12,74)$.

No ethnic predisposition has been reported, and most of the patients are young rural workers living in forested areas adjacent to rivers and streams. In these areas, some insects, including black fly (Simulium species), trigger the disease through insect saliva, leading to an immune reaction against Dsg1 through molecular mimicry $(4-7,9-12,74)$. This hypothesis is supported by high positivity rates of anti-Dsg1 IgG autoantibodies in the sera of healthy individuals living in endemic regions of fogo selvagem and the low prevalence of endemic PF in urbanized areas (4-7,9-12,74-76).

Pathophysiology and genetic factors. PF is mediated by autoantibodies against desmosomal proteins on the keratinocyte cell surface. The lesions in PF are induced by IgG (mainly IgG4 subclass) autoantibodies directed against Dsg1, a 160-kDa desmosomal cadherin transmembrane glycoprotein that mediates cell adhesion, expressed mainly in the granular layer of the epidermis (9-12,76). Dsg1 is closely associated with plakoglobin, an 85-kDa polypeptide found in the desmosomal plaques of keratinocytes, that links desmoglein to the intermediate keratin filament network inside the keratinocyte (76). Dsg1 is expressed more strongly in skin from the upper torso than that from the lower torso, buccal mucosa, or scalp, which may explain the distribution of lesions (7,10-12,74-76). The mechanism of acantholysis induction by specific autoantibodies may involve phosphorylation of intracellular proteins associated with desmosomes.

Other target antigens, including the acetylcholine receptor and desmoglein 3 (Dsg3), have been postulated to be relevant in the pathogenesis of PF (4-7,75-77). The regulation of keratinocyte cell-to-cell and cell-matrix adhesion is an important biological function of cutaneous acetylcholine and the progress in therapy of pemphigus using cholinergic drugs supports this concept $(7,9-12,74)$.

Patients with both sporadic and endemic forms of PF have anti-Dsg1 antibodies, their titer correlating with the extent and activity of the disease (76). The prevalence of anti-Dsg1 antibodies is high in people living in endemic areas of Brazil, and a Tunisian study found that anti-Dsg1 IgG antibodies were generally against pre-Dsg1 domains and/or C-terminals of Dsg1 (7,9-12,74-77). Some cases have been associated with the use of certain drugs, such as penicillamine (78). In patients who were treated with penicillamine, $\mathrm{PF}$ is more frequent than PV, with a ratio of $4: 1$. Penicillamine and captopril contain sulfhydryl groups that are speculated to interact with the sulfhydryl groups in Dsg1 and Dsg3 (7,12,76-78). Most patients 
with drug-induced pemphigus go into remission after the offending drug is discontinued.

Genetic factors predispose to the development of PF. The HLA-DRB1*04:01, HLA-DRB1*04:06, HLA-DRB1*14, HLA-DRB1*01:01, have been associated with a higher risk of PF (7,12,77-79). In the Brazilian population HLA-DRB1 alleles $* 04: 04, * 14: 02$, $* 14: 06$, and $* 01: 02$ have been reported as risk factors for fogo selvagem $(12,79)$. In France, people with DRB1*0102 and 0404 are at an increased risk of PF (77). It has been suggested that polymorphisms in the $2 q 33$ and $3 q 21$ chromosomal regions increase susceptibility to PF (80).

Clinical features. Unlike PV, PF only affects the skin. Mucosal lesions do not usually occur, because Dsg1 is only expressed in skin $(11,74)$. There are two versions of PF: an endemic version (fogo selvagem), and a localized version (pemphigus erythematosus or Senear-Usher syndrome), that typically share the same clinical findings (9-12,74-76).

Initial circumscribed lesions appear in seborrheic areas, such as the scalp, face, and chest (presternal and interscapular regions). Blisters appear slowly and are not obvious, because the cleavage is superficial, and small flaccid blisters break easily. The scales separate leaving painful erosions, surrounded by erythema and small vesicles along the edges (4-8,10-12,74-76). In the endemic version (fogo selvagem), the erosions are intensely painful, like 'wild fire', and predominantly affect young women in endemic regions $(8,74)$. In the localized version (pemphigus erythematosus/Senear-Usher syndrome), the lesions are similar to the malar erythema present in lupus erythematosus (strongly scaled erythematous plaques) that appear on sun-exposed areas such as the scalp, face and upper torso $(8,12,74-76,81)$. Pemphigus erythematosus mainly affects elderly patients, and medications, sun exposure and trauma are considered possible triggers $(8,80,81)$. In approximately $80 \%$ of these cases, immunoreactive deposits along the basement membrane and a mean titer of antinuclear antibodies can be detected, usually without the presence of anti-ds-DNA antibodies, which may suggest an association with lupus erythematosus $(8,80,81)$. In PF a common clinical finding is a positive Nikolsky sign, which is very specific in the diagnosis of pemphigus.

In the most severe form of PF, the skin lesions can dramatically progress, leading to exfoliative erythroderma, characterized by generalized erythema and diffuse scaling involving $90 \%$ or more of the cutaneous surface $(8,74)$. In cases of erythroderma of unknown origin, PF must be considered as a possible cause. These patients require prompt hospitalization to prevent serious and sometimes fatal complications from metabolic instability $(8,12,74-76)$.

Unusual presentations of PF have also been described, such as an acute rash with multiple hyperpigmented and hyperkeratotic lesions similar to seborrheic keratoses, lesions resembling impetigo, and scaly erythema on the scalp that may be confused with seborrheic dermatitis (74,80-82). In cases of sporadic PF in children, patients have the same primary lesions (blisters) and secondary lesions (erosions), but with a distinct configuration that has been described as arcuate, circinate and/or polycyclic (81-83). Pemphigus seborrhoicus is a special form of PF, with very superficial blisters, extensive erythematous plaques and erosions that develop in the seborrheic areas $(4,12)$.

Diagnosis. The diagnosis of PF is based on the following criteria: the overall clinical picture, including the patient's history and physical examination; the histopathological findings; the presence of autoantibodies as detected by direct and indirect immunofluorescence studies.

The histologic changes of pemphigus foliaceus, pemphigus erythematosus, and fogo selvagem are identical. The histopathological examination of early blisters demonstrates acantholysis of the upper epidermis, often resulting in a subcorneal cleft and leading to detachment of the epidermis in its midlevel. Subcorneal pustules contain neutrophils, fibrin and scattered acantholytic keratinocytes. The stratum corneum is often lost from the surface, the deeper epidermis usually remains intact, eosinophilic spongiosis and a mixed inflammatory infiltrate of neutrophils and eosinophils in the superficial dermis may be present $(4-7,12,74)$. These superficial blisters are histologically indistinguishable from those seen in staphylococcal scalded skin syndrome or bullous impetigo, because Dsg1 is targeted in both of these diseases, thus the histological features may not be diagnostic in the early stages (74-76). Chronic persistent lesions are acanthotic, papillomatous, and hyperkeratotic with focal parakeratosis. Dyskeratotic cells in the granular layer of older lesions distinguish PF from PV (4-7,12,74-76).

The DIF biopsy must be performed on the skin with a normal appearance, immediately adjacent to a lesion because inflamed and blistered skin can lead to the destruction of immune deposits $(28,29,74)$. DIF reveals IgG and C3 deposition in intercellular space staining (ICS), this model being called 'chicken wire' $(4,12,74-76)$. This is a result of the antibody bound to Dsg1 on desmosomes on the surface of keratinocyte cells. The intensity of this fluorescent stain in PF may be greater in the upper epidermis due to the increased density of Dsg1 $(28,74)$. IIF is positive in over $85 \%$ of $\mathrm{PF}$ cases and detects circulating IgG antibodies against epithelial cell surfaces, using monkey or guinea pig esophagus as substrate $(28-30,74)$. Staining of the IgG subclass for PF shows both IgG1 and IgG4 subclasses are produced against Dsg1, IgG4 being the predominant autoantibody subclass. IIF titers can be used to estimate the disease activity (28-30,74). ELISA detects anti-Dsg1 antibodies in $71 \%$ of patients with PF, using purified recombinant human Dsg1 to detect IgG autoantibodies in patient serum $(32-34,74,84)$. It was found that the sensitivity and specificity of detecting anti-Dsg1 antibodies by ELISA is $97.9 \%$, respectively $98.9 \%$ (84-86). In endemic regions with FS, ELISA specificity is relatively lower because more normal individuals in these areas test positive (false-positive increase) for total anti-Dsg1 IgG autoantibodies (85). ELISA titers have been found to correlate with disease activity, and are considered the best laboratory test for monitoring a patient's response to therapy (32-34,84-86). Trichoscopy has proven to be a useful tool in the differential diagnosis of scalp damage in pemphigus. Extravasations and yellow hemorrhagic crusts were the most common findings and the 'fried egg sign' (yellow dots with a whitish halo) was identified as a trichoscopy feature in pemphigus (87). 
The differential diagnosis of PF includes other forms of pemphigus, bullous impetigo, subcorneal pustular dermatosis, subacute cutaneous lupus erythematosus, and seborrheic dermatitis (74-76,84-86).

Treatment. The purpose of therapy in the handling of $\mathrm{PF}$ is to heal the existing lesions and to stop the surfacing of new ones. Before the advent of steroid therapy, PF was fatal in approximately $60 \%$ of patients, and almost always fatal in elderly patients with concurrent medical problems $(11,74-76,88)$. With corticosteroids, immunosuppressive therapy, and other therapeutic options, mortality has been dramatically reduced.

There are several factors to consider when deciding on a therapy such as the severity of the disease at introduction, associated medical illnesses in the likes of diabetes or tuberculosis, the patient's general health and age, hypertension, the speed of onset, efficacy, adverse effects, and the cost of the therapy $(4,10-12,74)$.

The initial treatment in PF is topical and oral corticosteroids. If the condition is not responsive to topical corticosteroid, systemic corticosteroid therapy may be initiated with prednisone at a dose of $0.5-1.5 \mathrm{mg} / \mathrm{kg}$ daily or prednisolone $20-40 \mathrm{mg}$ daily $(40,88)$. If there are no signs of remission in the first 2 weeks, a higher dose of prednisone is recommended. Nearly all patients reach total remission in 4-12 weeks, afterwards the dose of prednisone is reduced gradually. If no recurrence happens, the dose is maintained at 5 or $7.5 \mathrm{mg} / \mathrm{day}$, the reason being that low doses help prevent recurrences (88).

In patients who fail treatment with corticosteroids, have contraindications to systemic corticosteroids, or that have serious adverse effects, an immunosuppressant agent can be added, In cases of severe PF, an immunosuppressant and prednisone combined treatment can be used $(10-12,40,88)$. Immunosuppressants used include azathioprine, cyclophosphamide, and mycophenolate mofetil. Azathioprine (AZA) is a synthetic, quite potent, anti-inflammatory immunosuppressant. Thiopurine-methyltransferase dosing is required before the administration of AZA. The standard recommended dose is $1-3 \mathrm{mg} / \mathrm{kg} / \mathrm{day}(7,10-12,88)$. Mycophenolate mofetil (MMF) works by reducing the production of antibodies and inhibiting purine synthesis in stimulated $\mathrm{T}$ and $\mathrm{B}$ lymphocytes, blocking their proliferative response. The recommended dose of MMF is $1 \mathrm{~g} \times 2$ daily. It should be noted that the onset of action with MMF is slow and evidence of response occurs between 2 and 12 months of continued use (10-12,40,88). Cyclophosphamide (CP), an alkylating agent, is an immunosuppressive and cytotoxic drug that binds DNA regardless of the cell cycle phase. The dose of CP ranges from $1-3 \mathrm{mg} / \mathrm{kg}$ per day, generally given as 50-200 mg per day in doses equally divided or as a single dose in the morning $(10-12,40,88)$.

Other treatment options for refractory disease, or if there are contraindications to immunosuppressive agents include hydroxychloroquine $200 \mathrm{mg} x 2$ daily, dapsone $100 \mathrm{mg}$ daily or up to $1.5 \mathrm{mg} / \mathrm{kg}$ daily, methotrexate $10-20 \mathrm{mg}$ weekly, IVIg $2 \mathrm{~g} / \mathrm{kg}$ monthly, or RTX, given as 4 weekly infusions of $375 \mathrm{mg} / \mathrm{m}^{2}(10,11,47)$. Plasmapheresis and IVIg are therapeutic options in patients with recalcitrant disease $(44,45,88)$.

Considering the possible side effects of therapy, patients should be monitored closely. After the interruption of systemic corticosteroids in patients with total remission, adjuvant immunosuppression can be decreased over 6 to 12 months (41). The interruption of therapy depends on the clinical picture that shows no active cutaneous lesions over several months. Negative or low ELISA-Dsg1 values or negative immunofluorescence are useful to support the discontinuation of therapy (84-86).

Evolution and complications. PF tends to persist for months or years and is regarded as a benign disease that responds well to treatment. PF may be associated with thymoma, myasthenia gravis, lupus erythematosus, and other autoimmune bullous diseases $(88,89)$.

New cutaneous lesions, changes in primary morphology, rapid disease progression, constitutional symptoms, or failure to respond to appropriate therapies may suggest a concomitant viral skin infection, such as herpes simplex or cytomegalovirus (89).

\section{IgA pemphigus}

Epidemiology. IgA pemphigus is one of the rarest forms of the autoimmune blistering disease. The frequency of Ig A pemphigus is not well defined but has been reported in Asia (Japan and India), South America (Brazil), Europe (Scandinavian countries), and in the US (1,3,4-7,90). The distribution by sex and race is unknown, with cases being reported in all age groups, with a mean onset age of 53 years (90).

Pathophysiology and genetic factors. The exact pathomechanism of IgA pemphigus is not well defined, but it is related to Ig A autoantibodies that target desmosomal and non-desmosomal keratinocyte cell surface components. These components are cell-to-cell-adhering molecules, including Dsg1, Dsg3, and Dsc1 $(10-12,90,91)$.

Desmogleins and desmocollins are glycoproteins that belong to a superfamily of cadherin molecules. The subcorneal pustular dermatosis subtype exhibits IgA autoantibodies targeting the transmembrane glycoprotein Dsc1, while the antigen of the intraepidermal neutrophilic dermatosis has been found to interact with both Dsg1 and Dsg3 $(90,91)$.

In IgA pemphigus, autoantibodies bind to sites containing the monocyte/granulocyte IgA-Fc receptor (CD89), causing a massive inflammatory reaction and neutrophil infiltration of the epidermis, which clinically presents as blistering and pustule $(4,7,12,90)$. Although the targets of IgA antibodies have been identified, the direct pathogenic effects of the $\operatorname{Ig} \mathrm{A}$ autoantibodies and the exact mechanism of blister formation have not been established, thus a clinical picture of IgA pemphigus is not well known and requires additional investigations (90).

Clinical features. IgA pemphigus is a rare entity among the pemphigus diseases. It is considered to be a distinct entity that includes 2 clinical subtypes with different histologic features and different $\operatorname{Ig} \mathrm{A}$ deposition patterns in the epidermis: intraepidermal neutrophilic IgA dermatosis (IEN) and the subcorneal pustular dermatosis (SPD) $(4-7,10-12,90)$.

IgA pemphigus is characterized by fragile blisters and intraepidermal pustules or vesicles with neutrophilic infiltration in the erythematous skin located in flexural areas 
(axilla and groin), distal trunk, proximal limbs, and intertriginous sites. The lesions have a distinct tendency to coalesce, so that clinically often annular infiltrated plaques with accentuated margins and collarette-like scaling are seen. Mucosal involvement is rare $(4-8,10-12,90)$.

Diagnosis. Investigations in patients suspected of $\operatorname{Ig} \mathrm{A}$ pemphigus often include a skin biopsy to detect histological and immunological changes. Histologic examination of IgA pemphigus demonstrates subcorneal blisters with massive neutrophilic infiltration and with a mild loss of cohesion between keratinocytes. Histopathology is useful in differentiating the two major subtypes of IgA pemphigus $(4-7,12,90)$. In the SPD subtype, there are subcorneal pustules and increased intensity of $\operatorname{IgA}$ autoantibodies in the upper surface of the epidermis. In contrast, the IEN type is histologically characterized by suprabasal pustules and inflammatory infiltrates, located in the entire or lower part of the dermis $(7,12,90)$.

DIF is considered an early screening tool for the diagnosis of $\operatorname{Ig}$ A pemphigus, detecting the absence or presence of $\operatorname{Ig} \mathrm{A}$ autoantibodies on epidermal cell surfaces $(4,28-31,90)$. DIF can be used to differentiate IgA pemphigus from PF because the clinical differentiation between IgA pemphigus and PF is nearly impossible (90-92). DIF of PF identifies IgG autoantibodies against Dsg1 in contrast to the IgA deposits against Dsc1 found in IgA pemphigus $(29,90)$. Moreover, in contrast to IgA pemphigus, DIF in Sneddon-Wilkinson disease will be negative for IgA deposits against adhesion molecules, such as Dsc1, which is key in the diagnosis of the SPD subtype of IgA pemphigus (92).

IIF reveals circulating IgA antibodies in intraepidermal structures in half of the cases $(28,30,90)$. In the SPD type, Dsc1, one of the desmosomal cadherins, has been identified as a target autoantigen (91). In the IEN type, the autoantigen is not fully characterized, but there are several cases in which Dsg1 and Dsg3 have been demonstrated to be targets of the autoantibodies in this variant (12,90-93).

An association of $\operatorname{Ig}$ A pemphigus with monoclonal IgA gammopathies, multiple myeloma, HIV infection, Sjogren's syndrome, rheumatoid arthritis, and inflammatory bowel diseases (Crohn's disease, ulcerative colitis) have been reported $(90,94)$. While the direct relationship between these diseases and IgA pemphigus is still unclear, a thorough survey of hematologic and infectious disorders is advised for patients presenting with IgA pemphigus (90-92).

Differential diagnoses of IgA pemphigus include: classic subcorneal pustular dermatosis (Sneddon-Wilkinson disease), dermatitis herpetiformis, PF, eosinophilic pustular folliculitis, pemphigus herpetiformis and bacterial skin infections (90).

Treatment. The treatment for IgA pemphigus must be directed to reduce the inflammation because $\operatorname{IgA}$ pemphigus represents a group of autoimmune blistering skin diseases manifested clinically as chronic inflammation.

Generally, oral and topical corticosteroids with a suggested daily dose of $0.5-1 \mathrm{mg} / \mathrm{kg}$, are the mainstay of treatment for Ig A pemphigus (40,90-92). To minimize adverse effects, slow tapering of corticosteroids is advised in order to identify the lowest efficacious dose, and patients should be aware of the complications associated with long-term use of steroids, including osteoporosis, diabetes, cataracts, and infection (41). Many studies have found that dapsone may also be helpful in IgA pemphigus due to its antineutrophilic effects, but long-term treatment can determine hemolysis and methemoglobinemia $(95,96)$. Other drugs that have been reported to be successful in the treatment of $\operatorname{Ig}$ A pemphigus include colchicine, retinoids, mycophenolate mofetil, and adalimumab $(95,96)$. One case report described lesion regression after the addition of azithromycin to a local steroid and a keratolytic agent, while another case report described rapid response in SPD-type IgA pemphigus with oral isotretinoin treatment $(97,98)$.

Evolution and complications. IgA pemphigus presents as a milder and more limited disease, and by using appropriate treatment and follow-up, IgA pemphigus usually heals without scarring. Open wounds should be cared for in order to avoid infections and scarring. For patients diagnosed with $\operatorname{Ig} \mathrm{A}$ pemphigus, it is recommended that they undergo screening for hematological diseases, especially the elderly patients and those with systemic symptoms (90-93).

Complications that can occur during the disease are: infections (secondary to open wounds or drugs), malignancies (secondary to the chronic inflammatory process), growth retardation is possible secondary to medications used to treat IgA pemphigus during childhood (90-93).

\section{Conclusions}

The pathophysiology and autoantigen profile of bullous autoimmune diseases, especially pemphigus and its subforms, are more complex than previously assumed. Although the pathophysiology of blistering autoimmune diseases has been elucidated, there are still unanswered questions, including determination of the mechanism of the autoantibody production, or if there are any predictive factors of response to therapy. Pemphigus is a heterogeneous condition, and further studies are needed to assess the complexity of the disease.

As most patients require long-term immunosuppressive therapy, health care providers must establish effective and interdisciplinary management of the side effects of therapy.

The treatment of pemphigus should target the cells, autoantibodies, and/or factors directly involved in pathogenesis to avoid general immune suppression. New treatments, including B-cell-directed therapy, are the new therapeutic frontier for this kind of disease.

In this review, we summarized the process of establishing and revising the diagnostic criteria, and the clinical and therapeutic aspects of the main types of intraepidermal blistering diseases from the pemphigus group.

\section{Acknowledgements}

Professional editing, linguistic and technical assistance performed by Irina Radu, Individual Service Provider.

\section{Funding}

No funding was received. 


\section{Availability of data and materials}

All information provided in this review is documented by relevant references.

\section{Authors' contributions}

VVC and MPT conceived and supervised the research. CP, MFH and EPA analyzed the literatue data. VVC, CP, MFH, EPA and MPT contributed to data acquisition and interpretation and wrote the manuscript. All authors contributed to acquisition, analysis and systematization of the literature data, manuscript writing and critical revision of it for important intellectual content. All authors reviewed the results and read and approved the final version of the manuscript.

\section{Ethics approval and consent to participate}

Not applicable.

\section{Patient consent for publication}

Consent was provided by the patient to use any clinical image that did not reveal personal identity.

\section{Competing interests}

The authors declare that they have no competing interests and they have no financial relationships to disclose.

\section{References}

1. Lakoš Jukić I, Jerković Gulin S and Marinović B: Blistering diseases in the mature patient. Clin Dermatol 36: 231-238, 2018.

2. Kasperkiewicz M: COVID-19 outbreak and autoimmune bullous diseases: A systematic review of published cases. J Am Acad Dermatol 84: 563-568, 2021.

3. Chaudhari P and Marinkovich MP: What's new in blistering disorders? Curr Allergy Asthma Rep 7: 255-263, 2007.

4. Hofmann SC, Juratli HA and Eming R: Bullous autoimmune dermatoses. J Dtsch Dermatol Ges 16: 1339-1358, 2018.

5. Bickle K, Roark TR and Hsu S: Autoimmune bullous dermatoses: A review. Am Fam Physician 65: 1861-1870, 2002.

6. Patrício P, Ferreira C, Gomes MM and Filipe P: Autoimmune bullous dermatoses: A review. Ann NY Acad Sci 1173: 203-210, 2009.

7. Egami S, Yamagami J and Amagai M: Autoimmune bullous skin diseases, pemphigus and pemphigoid. J Allergy Clin Immunol 145: 1031-1047, 2020.

8. Kneisel A and Hertl M: Autoimmune bullous skin diseases. Part 1: Clinical manifestations. J Dtsch Dermatol Ges 9: 844-856; quiz 857, 2011 (In English, German).

9. Kasperkiewicz M, Ellebrecht CT, Takahashi H, Yamagami J, Zillikens D, Payne AS and Amagai M: Pemphigus. Nat Rev Dis Primers 3: 17026, 2017.

10. Pollmann R, Schmidt T, Eming R and Hertl M: Pemphigus: A comprehensive review on pathogenesis, clinical presentation and novel therapeutic approaches. Clin Rev Allergy Immunol 54: $1-25,2018$.

11. Melchionda V and Harman KE: Pemphigus vulgaris and pemphigus foliaceus: An overview of the clinical presentation, investigations and management. Clin Exp Dermatol 44: 740-746, 2019.

12. Didona D, Maglie R, Eming R and Hertl M: Pemphigus: Current and future therapeutic strategies. Front Immunol 10:1418, 2019.

13. Oktarina DA, van der Wier G, Diercks GF, Jonkman MF and Pas HH: IgG-induced clustering of desmogleins 1 and 3 in skin of patients with pemphigus fits with the desmoglein nonassembly depletion hypothesis. Br J Dermatol 165: 552-562, 2011.
14. Amber KT, Valdebran M and Grando SA: Non-desmoglein antibodies in patients with pemphigus vulgaris. Front Immunol 9: 1190, 2018

15. Yan L, Wang JM and Zeng K: Association between HLA-DRB1 polymorphisms and pemphigus vulgaris: A meta-analysis. $\mathrm{Br}$ J Dermatol 167: 768-777, 2012.

16. Sultan AS, Villa A, Saavedra AP, Treister NS and Woo SB: Oral mucous membrane pemphigoid and pemphigus vulgaris-a retrospective two-center cohort study. Oral Dis 23: 498-504, 2017.

17. Mustafa MB, Porter SR, Smoller BR and Sitaru C: Oral mucosal manifestations of autoimmune skin diseases. Autoimmun Rev 14: 930-951, 2015

18. Rashid H, Lamberts A, Diercks GFH, Pas HH, Meijer JM, Bolling MC and Horváth B: Oral lesions in autoimmune bullous diseases: An Overview of clinical characteristics and diagnostic algorithm. Am J Clin Dermatol 20: 847-861, 2019.

19. Carvalho AA, Santos Neto DAD, Carvalho MADR, Eleutério SJP and Xavier AREO: Neonatal pemphigus in an infant born to a mother with pemphigus vulgaris: A case report. Rev Paul Pediatr 37: 130-134, 2019.

20. Panko J, Florell SR, Hadley J, Zone J, Leiferman K and Vanderhooft S: Neonatal pemphigus in an infant born to a mother with serologic evidence of both pemphigus vulgaris and gestational pemphigoid. J Am Acad Dermatol 60: 1057-1062, 2009.

21. Amer YB and Al Ajroush W: Pemphigus vulgaris in a neonate. Ann Saudi Med 27: 453-455, 2007.

22. Huang YH, Wang SH, Kuo TT and Chi CC: Pemphigus vegetans occurring in a split-thickness skin graft. Dermatol Surg 31: 240-243, 2005.

23. Sillevis Smitt JH, Mulder TJ, Albeda FW and Van Nierop JC: Pemphigus vegetans in a child. Br J Dermatol 127: 289-291, 1992.

24. Jain V, Jindal $\mathrm{N}$ and Imchen $\mathrm{S}$ : Localized pemphigus vegetans without mucosal involvement. Indian J Dermatol 59: 210, 2014.

25. Son YM, Kang HK, Yun JH, Roh JY and Lee JR: The Neumann type of pemphigus vegetans treated with combination of dapsone and steroid. Ann Dermatol 23 (Suppl 3): S310-S313, 2011.

26. Peterman CM, Vadeboncoeur S, Schmidt BA and Gellis SE: Pediatric pemphigus herpetiformis: Case report and review of the literature. Pediatr Dermatol 34: 342-346, 2017.

27. Shimizu Y, Wakabayashi K, Hayashi Y, Hara K, Aoyama R, Niimi T, Tomino Y, Wada R, Hata M and Suzuki Y: MPGN type 3 associated with pemphigus herpetiformis mimicking PGNMID and dermatitis herpetiformis. Case Rep Nephrol Dial 9: 15-24, 2019.

28. Mihai S and Sitaru C: Immunopathology and molecular diagnosis of autoimmune bullous diseases. J Cell Mol Med 11 462-481, 2007.

29. Aoki V, Sousa JX Jr, Fukumori LM, Périgo AM, Freitas EL and Oliveira ZN: Direct and indirect immunofluorescence. An Bras Dermatol 85: 490-500, 2010 (In English, Portuguese).

30. Mihályi L, Kiss M, Dobozy A, Kemény L and Husz S: Clinical relevance of autoantibodies in patients with autoimmune bullous dermatosis. Clin Dev Immunol 2012: 369546, 2012.

31. Saschenbrecker S, Karl I, Komorowski L, Probst C, Dähnrich C, Fechner K, Stöcker W and Schlumberger W: Serological diagnosis of autoimmune bullous skin diseases. Front Immunol 10: 1974, 2019.

32. Abasq C, Mouquet H, Gilbert D, Tron F, Grassi V, Musette P and Joly P: ELISA testing of anti-desmoglein 1 and 3 antibodies in the management of pemphigus. Arch Dermatol 145: 529-535, 2009.

33. Mortazavi H, Shahdi M, Amirzargar AA, Naraghi ZS, Valikhani M, Daneshpazhooh M, Vasheghani-Farahani A, Sedaghat $\mathrm{M}$ and Chams-Davatchi C: Desmoglein ELISA in the diagnosis of pemphigus and its correlation with the severity of pemphigus vulgaris. Iran J Allergy Asthma Immunol 8: 53-56, 2009.

34. Tampoia M, Giavarina D, Di Giorgio C and Bizzaro N: Diagnostic accuracy of enzyme-linked immunosorbent assays (ELISA) to detect anti-skin autoantibodies in autoimmune blistering skin diseases: A systematic review and meta-analysis. Autoimmun Rev 12: 121-126, 2012

35. Naseer SY, Seiffert-Sinha K and Sinha AA: Detailed profiling of anti-desmoglein autoantibodies identifies anti-Dsg1 reactivity as a key driver of disease activity and clinical expression in pemphigus vulgaris. Autoimmunity 48: 231-241, 2015. 
36. Di Zenzo G, Di Lullo G, Corti D, Calabresi V, Sinistro A, Vanzetta F, Didona B, Cianchini G, Hertl M, Eming R, et al: Pemphigus autoantibodies generated through somatic mutations target the desmoglein-3 cis-interface. J Clin Invest 122: 3781-3790, 2012.

37. Lakshmi MJD, Jaisankar TJ, Rajappa M, Thappa DM, Chandrashekar L, Divyapriya D, Munisamy M and Revathy G: Correlation of antimuscarinic acetylcholine receptor antibody titers and antidesmoglein antibody titers with the severity of disease in patients with pemphigus. J Am Acad Dermatol 76: 895-902, 2017.

38. Heelan K, Mahar AL, Walsh S and Shear NH: Pemphigus and associated comorbidities: A cross-sectional study. Clin Exp Dermatol 40: 593-599, 2015.

39. Parameswaran A, Attwood K, Sato R, Seiffert-Sinha K and Sinha AA: Identification of a new disease cluster of pemphigus vulgaris with autoimmune thyroid disease, rheumatoid arthritis and type I diabetes. Br J Dermatol 172: 729-738, 2015.

40. Almugairen N,Hospital V,Bedane C, Duvert-Lehembre S, Picard D, Tronquoy AF, Houivet E, D'incan M and Joly P: Assessment of the rate of long-term complete remission off therapy in patients with pemphigus treated with different regimens including medium- and high-dose corticosteroids. J Am Acad Dermatol 69: 583-588, 2013.

41. Satyanarayanasetty D, Pawar K, Nadig P and Haran A: Multiple adverse effects of systemic corticosteroids: A case report. J Clin Diagn Res 9: FD01-FD2, 2015.

42. Ahmed AR, Kaveri S and Spigelman Z: Long-term remissions in recalcitrant pemphigus vulgaris. N Engl J Med 373: 2693-2694 2015.

43. Beissert S, Werfel T, Frieling U, Böhm M, Sticherling M, Stadler R, Zillikens D, Rzany B, Hunzelmann N, Meurer M, et al: A comparison of oral methylprednisolone plus azathioprine or mycophenolate mofetil for the treatment of pemphigus. Arch Dermatol 142: 1447-1454, 2006.

44. Amagai M, Ikeda S, Shimizu H, Iizuka H, Hanada K, Aiba S, Kaneko F, Izaki S, Tamaki K, Ikezawa Z, et al: Pemphigus study group. A randomized double-blind trial of intravenous immunoglobulin for pemphigus. J Am Acad Dermatol 60: 595-603, 2009

45. Higashihara T, Kawase M, Kobayashi M, Hara M, Matsuzaki H, Uni R, Matsumura M, Etoh T and Takano H: Evaluating the efficacy of double-filtration plasmapheresis in treating five patients with drug-resistant pemphigus. Ther Apher Dial 21: 243-247, 2017.

46. Eming $\mathrm{R}$ and Hertl $\mathrm{M}$ : Immunoadsorption in pemphigus. Autoimmunity 39: 609-616, 2006.

47. Du FH, Mills EA and Mao-Draayer Y: Next-generation anti-CD20 monoclonal antibodies in autoimmune disease treatment. Auto Immun Highlights 8: 12, 2017.

48. Wang HH, Liu CW, Li YC and Huang YC: Efficacy of rituximab for pemphigus: A systematic review and meta-analysis of different regimens. Acta Derm Venereol 95: 928-932, 2015

49. Seyfizadeh N, Seyfizadeh N, Hasenkamp J and Huerta-Yepez S: A molecular perspective on rituximab: A monoclonal antibody for B cell non Hodgkin lymphoma and other affections. Crit Rev Oncol Hematol 97: 275-290, 2016.

50. Ahmed AR, Spigelman Z, Cavacini LA and Posner MR: Treatment of pemphigus vulgaris with rituximab and intravenous immune globulin. N Engl J Med 355: 1772-1779, 2006.

51. Schmidt E, Goebeler M and Zillikens D: Rituximab in severe pemphigus. Ann N Y Acad Sci 1173: 683-691, 2009.

52. Chiu HY, Chang CY, Hsiao CH and Wang LF: Concurrent cytomegalovirus and herpes simplex virus infection in pemphigus vulgaris treated with rituximab and prednisolone. Acta Derm Venereol 93: 200-201, 2013.

53. Wei KC, Chen W, Tang PL and Huang YT: Pneumocystis jirovecii pneumonia infection in pemphigus patients treated with rituximab: An observational nationwide epidemiological study in Taiwan. Eur J Dermatol 28: 713-715, 2018.

54. Amber KT, Kodiyan J, Bloom R and Hertl M: The controversy of hepatitis $\mathrm{C}$ and rituximab: A multidisciplinary dilemma with implications for patients with pemphigus. Indian J Dermatol Venereol Leprol 82: 182-183, 2016.

55. Frampton JE: Rituximab: A review in pemphigus vulgaris. Am J Clin Dermatol 21: 149-156, 2020.

56. Rapp M, Pentland A and Richardson C: Successful treatment of pemphigus vulgaris with ofatumumab. J Drugs Dermatol 17: $1338-1339,2018$

57. Ellebrecht CT, Choi EJ, Allman DM, Tsai DE, Wegener WA, Goldenberg DM and Payne AS: Subcutaneous veltuzumab, a humanized anti-CD20 antibody, in the treatment of refractory pemphigus vulgaris. JAMA Dermatol 150: 1331-1335, 2014.
58. Vinay K, Kanwar AJ, Mittal A, Dogra S, Minz RW and Hashimoto T: Intralesional rituximab in the treatment of refractory oral pemphigus vulgaris. JAMA Dermatol 151: 878-882, 2015.

59. Paolino G, Didona D, Magliulo G, Iannella G, Didona B, Raffaele S, Moliterni E, Donati M, Ciofalo A, Granata G, et al: Paraneoplastic pemphigus: Insight into the autoimmune pathogenesis, clinical features and therapy. Int J Mol Sci 18: 2532, 2017.

60. Tsuchisaka A, Numata S, Teye K, Natsuaki Y, Kawakami T, Takeda Y, Wang W, Ishikawa K, Goto M, Koga H, et al: Epiplakin is a paraneoplastic pemphigus autoantigen and related to bronchiolitis obliterans in Japanese patients. J Investig Dermatol 136: 399-408, 2016.

61. Czernik A, Camilleri M, Pittelkow MR and Grando SA: Paraneoplastic autoimmune multiorgan syndrome: 20 years after. Int J Dermatol 50: 905-914, 2011.

62. Wade MS and Black MM: Paraneoplastic pemphigus: A brief update. Australas J Dermatol 46: 1-8; quiz 9-10, 2005.

63. Martel P, Loiseau P, Joly P, Busson M, Lepage V, Mouquet H, Courville $\mathrm{P}$, Flageul $\mathrm{B}$, Charron $\mathrm{D}$, Musette $\mathrm{P}$, et al: Paraneoplastic pemphigus is associated with the DRB $1 * 03$ allele. J Autoimmun 20: 91-95, 2003.

64. Kim JH and Kim SC: Paraneoplastic pemphigus: Paraneoplastic autoimmune disease of the skin and mucosa. Front Immunol 10 1259, 2019.

65. Healy WJ, Peters S and Nana-Sinkam SP: A middle-aged man presenting with unexplained mucosal erosions and progressive dyspnoea. BMJ Case Rep 2015: bcr2014208677, 2015.

66. Mar WA, Glaesser R, Struble K, Stephens-Groff S, Bangert J and Hansen RC: Paraneoplastic pemphigus with bronchiolitis obliterans in a child. Pediatr Dermatol 20: 238-242, 2003.

67. Wieczorek M and Czernik A: Paraneoplastic pemphigus: A short review. Clin Cosmet Investig Dermatol 9: 291-295, 2016.

68. Cummins DL, Mimouni D, Tzu J, Owens N, Anhalt GJ and Meyerle JH: Lichenoid paraneoplastic pemphigus in the absence of detectable antibodies. J Am Acad Dermatol 56: 153-159, 2007.

69. Hashimoto T, Amagai M, Watanabe K, Chorzelski TP, Bhogal BS, Black MM, Stevens HP, Boorsma DM, Korman NJ, Gamou S, et al: Characterization of paraneoplastic pemphigus autoantigens by immunoblot analysis. J Invest Dermatol 104: 829-834, 1995.

70. Frew JW and Murrell DF: Current management strategies in paraneoplastic pemphigus (paraneoplastic autoimmune multiorgan syndrome). Dermatol Clin 29: 607-612, 2011.

71. Lee A, Sandhu S, Imlay-Gillespie L, Mulligan S and Shumack S: Successful use of Bruton's kinase inhibitor, ibrutinib, to control paraneoplastic pemphigus in a patient with paraneoplastic autoimmune multiorgan syndrome and chronic lymphocytic leukaemia. Australas J Dermatol 58: e240-e242, 2017.

72. Hohwy T, Bang K, Steiniche T, Peterslund NA and d'Amore F: Alemtuzumab-induced remission of both severe paraneoplastic pemphigus and leukaemic bone marrow infiltration in a case of treatment-resistant B-cell chronic lymphocytic leukaemia. Eur J Haematol 73: 206-209, 2004

73. Gu L and Ye S: Tocilizumab cannot prevent the development of bronchiolitis obliterans in patients with Castleman disease-associated paraneoplastic pemphigus. J Clin Rheumatol 25: e77-e78, 2019.

74. James KA, Culton DA and Diaz LA: Diagnosis and clinical features of pemphigus foliaceus. Dermatol Clin 29: 405-412, viii, 2011.

75. Bastuji-Garin S, Souissi R, Blum L, Turki H, Nouira R, Jomaa B, Zahaf A, Ben Osman A, Mokhtar I, Fazaa B, et al: Comparative epidemiology of pemphigus in Tunisia and France: Unusual incidence of pemphigus foliaceus in young Tunisian women. J Invest Dermatol 104: 302-305, 1995.

76. Shirakata Y, Amagai M, Hanakawa Y, Nishikawa T and Hashimoto $\mathrm{K}$ : Lack of mucosal involvement in pemphigus foliaceus may be due to low expression of desmoglein 1. J Invest Dermatol 110: 76-78, 1998

77. Abida O, Kallel-Sellami M, Joly P, Ben Ayed M, Zitouni M, Masmoudi A, Mokni M, Fezzaa B, Ben Osman A, Kammoun MR, et al: Franco-Tunisian group of survey and research on pemphigus. Anti-desmoglein 1 antibodies in healthy related and unrelated subjects and patients with pemphigus foliaceus in endemic and non-endemic areas from Tunisia. J Eur Acad Dermatol Venereol 23: 1073-1078, 2009.

78. Matkaluk RM and Bailin PL: Penicillamine-induced pemphigus foliaceus. A fatal outcome. Arch Dermatol 117: 156-157, 1981. 
79. Aoki V, Millikan RC, Rivitti EA, Hans-Filho G, Eaton DP, Warren SJ, Li N, Hilario-Vargas J, Hoffmann RG and Diaz LA Cooperative Group for Fogo Selvagem Research: Environmental risk factors in endemic pemphigus foliaceus (fogo selvagem). J Investig Dermatol Symp Proc 9: 34-40, 2004.

80. Dalla-Costa R, Pincerati MR, Beltrame MH, Malheiros D and Petzl-Erler ML: Polymorphisms in the 2q33 and 3q21 chromosome regions including $\mathrm{T}$-cell coreceptor and ligand genes may influence susceptibility to pemphigus foliaceus. Hum Immunol 71: 809-817, 2010.

81. Bruckner N, Katz RA and Hood AF: Pemphigus foliaceus resembling eruptive seborrheic keratoses. Arch Dermatol 116: 815-816, 1980.

82. Jacyk WK and Simson IW: Pemphigus erythematosus resembling multiple seborrheic keratoses. Arch Dermatol 126: 543-544, 1990.

83. Metry DW, Hebert AA and Jordon RE: Nonendemic pemphigus foliaceus in children. J Am Acad Dermatol 46: 419-422, 2002.

84. Gomi H, Kawada A, Amagai M and Matsuo I: Pemphigus erythematosus: Detection of anti-desmoglein-1 antibodies by ELISA. Dermatology 199: 188-189, 1999.

85. Qaqish BF, Prisayanh P, Qian Y, Andraca E, Li N, Aoki V, Hans-Filho G, dos Santos V, Rivitti EA and Diaz LA; Cooperative Group on Fogo Selvagem Research: Development of an IgG4-based predictor of endemic pemphigus foliaceus (fogo selvagem). J Invest Dermatol 129: 110-118, 2009.

86. Schmidt E, Dähnrich C, Rosemann A, Probst C, Komorowski L, Saschenbrecker S, Schlumberger W, Stöcker W, Hashimoto T, Bröcker EB, et al: Novel ELISA systems for antibodies to desmoglein 1 and 3: Correlation of disease activity with serum autoantibody levels in individual pemphigus patients. Exp Dermatol 19: 458-463, 2010.

87. Sar-Pomian M, Kurzeja M, Rudnicka L and Olszewska M: The value of trichoscopy in the differential diagnosis of scalp lesions in pemphigus vulgaris and pemphigus foliaceus. An Bras Dermatol 89: 1007-1012, 2014.

88. Sami N, Qureshi A and Ahmed AR: Steroid sparing effect of intravenous immunoglobulin therapy in patients with pemphigus foliaceus. Eur J Dermatol 12: 174-178, 2002.

89. Sawamura S, Kajihara I, Makino K, Makino T, Fukushima S, Jinnin M, Oyama B, Hashimoto T and Ihn H: Systemic lupus erythematosus associated with myasthenia gravis, pemphigus foliaceus and chronic thyroiditis after thymectomy. Australas J Dermatol 58: e120-e122, 2017.
90. Kridin K, Patel PM, Jones VA, Cordova A and Amber KT: IgA pemphigus: A systematic review. J Am Acad Dermatol 82: 1386-1392, 2020.

91. Düker I, Schaller J, Rose C, Zillikens D, Hashimoto T and Kunze J: Subcorneal pustular dermatosis-type IgA pemphigus with autoantibodies to desmocollins 1, 2, and 3. Arch Dermatol 145: 1159-1162, 2009.

92. Watts PJ and Khachemoune A: Subcorneal pustular dermatosis: A review of 30 years of progress. Am J Clin Dermatol 17: 653-671, 2016.

93. Kuan YZ, Chiou HT, Chang HC, Chan HL and Kuo TT: Intraepidermal neutrophilic IgA dermatosis. J Am Acad Dermatol 22: 917-919, 1990

94. Adam Z, Krejcí M, Pour L, Feit J, Büchler T and Hájek R: IgA pemphigus associated with monoclonal gammopathy completely resolved after achievement of complete remission of multiple myeloma with bortezomib, cyclophosphamide and dexamethasone regimen. Wien Klin Wochenschr 122: 311-314, 2010.

95. Hirata Y, Abe R, Kikuchi K, Hamasaka A, Shinkuma S, Ujiie H, Nomura T, Nishie W, Arita K and Shimizu H: Intraepidermal neutrophilic IgA pemphigus successfully treated with dapsone. Eur J Dermatol 22: 282-283, 2012.

96. Howell SM, Bessinger GT, Altman CE and Belnap CM: Rapid response of $\operatorname{IgA}$ pemphigus of the subcorneal pustular dermatosis subtype to treatment with adalimumab and mycophenolate mofetil. J Am Acad Dermatol 53: 541-543, 2005.

97. Bliziotis I, Rafailidis P, Vergidis P and Falagas ME: Regression of subcorneal pustular dermatosis type of IgA pemphigus lesions with azithromycin. J Infect 51: E31-E34, 2005.

98. Gruss C, Zillikens D, Hashimoto T, Amagai M, Kroiss M, Vogt T, Landthaler M and Stolz W: Rapid response of IgA pemphigus of subcorneal pustular dermatosis type to treatment with isotretinoin. J Am Acad Dermatol 43: 923-926, 2000.

This work is licensed under a Creative Commons Attribution-NonCommercial-NoDerivatives 4.0 International (CC BY-NC-ND 4.0) License. 